\title{
OPEN Nanovibrational stimulation inhibits osteoclastogenesis and enhances osteogenesis in co-cultures
}

\begin{abstract}
John W. Kennedy ${ }^{1 \bowtie}$, P. Monica Tsimbouri ${ }^{1}$, Paul Campsie ${ }^{2}$, Shatakshi Sood ${ }^{3}$, Peter G. Childs ${ }^{2}$, Stuart Reid ${ }^{2}$, Peter S. Young ${ }^{1}$, Dominic R. M. Meek ${ }^{4}$, Carl S. Goodyear ${ }^{3}$ \& Matthew J. Dalby ${ }^{1}$

Models of bone remodelling could be useful in drug discovery, particularly if the model is one that replicates bone regeneration with reduction in osteoclast activity. Here we use nanovibrational stimulation to achieve this in a 3D co-culture of primary human osteoprogenitor and osteoclast progenitor cells. We show that $1000 \mathrm{~Hz}$ frequency, $40 \mathrm{~nm}$ amplitude vibration reduces osteoclast formation and activity in human mononuclear $\mathrm{CD} 14^{+}$blood cells. Additionally, this nanoscale vibration both enhances osteogenesis and reduces osteoclastogenesis in a co-culture of primary human bone marrow stromal cells and bone marrow hematopoietic cells. Further, we use metabolomics to identify Akt (protein kinase $C$ ) as a potential mediator. Akt is known to be involved in bone differentiation via transforming growth factor beta 1 (TGF $\beta 1$ ) and bone morphogenetic protein 2 (BMP2) and it has been implicated in reduced osteoclast activity via Guanine nucleotide-binding protein subunit $\alpha 13$ (G $\alpha 13)$. With further validation, our nanovibrational bioreactor could be used to help provide humanised 3D models for drug screening.
\end{abstract}

Osteoporosis represents a large unmet clinical need where normal bone homeostasis is disrupted, leading to reduced bone density and increased fragility ${ }^{1}$. This has major detrimental effects on the quality of life of an increasingly ageing population. Osteoporosis disproportionately affects women ${ }^{1-3}$ and fragility fractures arising from osteoporosis are a large socioeconomic burden, with an estimated cost of $£ 4.4$ billion per annum in the UK and $\$ 20$ billion per annum in the $\mathrm{US}^{3,4}$.

Approximately $10 \%$ of total bone mass of an adult is remodelled each year, and varies by anatomical location ${ }^{5}$. Osteoporosis occurs due to a breakdown in the normal balanced, bone homeostasis process of formation and resorption. Treatment focuses on the use of agents such as bisphosphonates or denosumab to reduce osteoclastrelated bone resorption ${ }^{6}$. However, more consideration needs to be directed to the mesenchymal derived osteoblast population given that it is bone formation that is reduced and limiting osteoclast formation is only slowing the disease rather than reversing it $^{7}$. It is notable that targeting osteogenesis will also influence osteoclast regulation. This is because osteoblastic cells signal to macrophages to fuse and form osteoclasts via receptor activator of nuclear factor $\kappa \mathrm{B}$ ligand (RANKL) and macrophage-colony stimulating factor (M-CSF), or prevent fusion by expression of the RANKL decoy receptor osteoprotegrin (OPG), ultimately providing homeostatic control ${ }^{8}$.

Due to the co-dependence and dynamic balance between bone forming and bone resorbing cells, reliance on overly simplified mono-culture cell lines, for example MG63 and RAW264.7 macrophage derived osteoclasts, may not be appropriate for use in drug discovery pipelines. This can further slow the development of new therapeutics 9 . Non-human animal models for osteoporosis are useful but, again, limited. Well used models do exist, for example, ovariectomy to model postmenopausal osteoporosis and sciatic neurectomy to model disuse osteoporosis ${ }^{10}$. However, rodents are the most common model and they lack the Haversian remodelling system found in humans ${ }^{11}$. Furthermore, only humans and primates naturally suffer from osteoporosis ${ }^{11}$. Thus, improved co-cultures based on human cells could be a useful tool in osteoporosis and bone homeostasis research.

\footnotetext{
${ }^{1}$ Centre for the Cellular Microenvironment, Institute of Molecular, Cell and Systems Biology, College of Medical, Veterinary and Life Sciences, University of Glasgow, Glasgow G12 800, UK. '2SUPA Department of Biomedical Engineering, University of Strathclyde, Glasgow G1 1OE, UK. ${ }^{3}$ Institute of Infection, Immunity and Inflammation, Glasgow Biomedical Research Centre, University Place, University of Glasgow, Glasgow G12 8TA, UK. ${ }^{4}$ Department of Trauma and Orthopaedics, Queen Elizabeth University Hospital, Glasgow G51 4TF, UK. $\square$ email: ian.kennedy4@ nhs.scot
} 
To meet this need, co-cultures are being developed. Most co-cultures are based on seeding both osteoblastic and osteoclastic cell simultaneously but are generally established using murine cells or immortalised cell lines rather than primary human cells that more accurately reflect the in vivo human phenotype ${ }^{12-16}$. We have previously reported a simple co-culture system comprising of primary bone marrow stromal cells (BMSCs) and bone marrow hematopoietic cells (BMHCs) where osteogenic and osteoclastic development can be observed and their interactions studied ${ }^{17,18}$. This co-culture system was employed in this new study to test the hypothesis that nanovibrations can be used to simultaneously drive osteogenesis and reduce osteoclastogenesis.

We have recently reported a nanovibrational bioreactor that delivers $30-40 \mathrm{~nm}$ vertical displacements to cell cultures at $1000 \mathrm{~Hz}^{19-21}$. This nanomechanical stimulus drives osteospecific differentiation of BMSCs in 2D and 3D (hydrogel) cultures without need for factors such as dexamethasone, or bone morphogenetic protein 2 (BMP-2), a potent osteoinducer used in clinical practice ${ }^{19-21}$. In this new study, we reproduce these conditions reported as being optimal for osteogenesis ${ }^{21,22}$. The technique could be beneficial in helping understand osteogenesis without recourse to chemical induction and has already illustrated the roles of mechanotransductive cation channels such as TRPV1 (transient receptor potential cation channel subfamily V member 1) and piezo 1 and $2^{20}$. Additionally, nanovibration has been found to produce therapeutic levels of ROS, inflammation and balancing pathways ${ }^{22}$.

To test the effect of nanovibration, we utilised an osteoclast-forming monoculture (primary human CD14 ${ }^{+}$ cells isolated from peripheral blood mononuclear cells, PBMCs) and our primary human BMSC/BMHC coculture. In addition, we performed analysis in $2 \mathrm{D}$ and in $3 \mathrm{D}$ using collagen hydrogels in acknowledgement that bone is a $3 \mathrm{D}$ tissue.

The nanovibrational bioreactor. The bioreactor uses the reverse piezoelectric effect to produce mechanical expansions from applied voltages. Piezo actuators are attached to an aluminium base block; this mass ensures that the expansion is upwards, into the cell culture. The piezo ceramics are then glued/bolted to a ferrous top plate. This allows attachment of cell culture plastics with soft magnets. The magnets are attached to the base of the culture plates and then magnetically coupled to the bioreactor top plate. This allows easy removal of the cultureware for ease of maintenance (e.g. media changes). A power supply is used to deliver the $1000 \mathrm{~Hz}$ sine wave signal with a pre-determined voltage to achieve an expansion (Fig. 1a) ${ }^{21}$.

Laser interferometry was used to measure the nanometric displacements in 2D and 3D (collagen hydrogel) culture. To achieve this, prismatic tape was placed into the wells or on top of collagen hydrogels $(0.8 \mathrm{mg} / \mathrm{ml} \mathrm{rat} \mathrm{tail}$ collagen) cast into the wells of 24 well plates (Fig. 1b,c) ${ }^{22,23}$. The collagen has low stiffness, $E=\sim 25$ Pa measured by parallel plate rheology; well below that required to stimulate osteogenesis of MSCs $(30-40 \mathrm{kPa})^{24}$. This ensures that while the gel is biocompatible, it is the nanovibrations that drive any osteogenesis. In $2 \mathrm{D}$ conditions with two 24 well plates magnetically coupled to the bioreactor top plate, an average displacement of $40.6 \mathrm{~nm}$ was noted (Fig. 1d). In 3D conditions with a single 24 well plate magnetically coupled, an average displacement of $44.4 \mathrm{~nm}$ was noted (Fig. 1e; please note that individual well vibrations are presented in Supplementary Fig. 1). Thus, we establish that the bioreactor can generate precise nanoscale vibrations in both $2 \mathrm{D}$ and $3 \mathrm{D}$ culture systems. We note that within the confines of a cell culture plate well water is incompressible ${ }^{25}$ and so acts as a solid object when vibrated. Equally, hydrogels, such as collagen gels, are mainly water and also transfer the mechanical motion of the vibration with little alteration of amplitude.

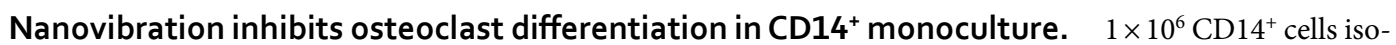
lated from PMBCs obtained from buffy coats were cultured in 2D culture in 24 well plates with $40 \mathrm{~nm}$ nanovibrational stimulation for up to 7 days. Non-stimulated controls were also used and all cultures (stimulated and control) were supplemented with $25 \mathrm{ng} / \mathrm{ml}$ of human M-CSF and $25 \mathrm{ng} / \mathrm{ml}$ of human RANKL in order to permit monocyte fusion and osteoclastogenesis. Alamar blue reduction was used to infer viability and showed that nanovibrational culture had no detrimental effect on the CD14 ${ }^{+}$cultures (Fig. 2a). The number of multinucleated osteoclasts per well after 7 days of culture was reduced (Fig. 2b), as was the average size of the osteoclasts formed (Fig. 2c). Figure 2d shows typical TRAP (tartrate resistant acid phosphatase, a marker of osteoclast formation $)^{13}$ images used in quantitative analysis (control top, $1000 \mathrm{~Hz}$ stimulated bottom). Together, this infers that the monocytes seeded into the nanovibrational cultures remain viable but undergo fusion into osteoclasts less frequently. SEM (scanning electron microscopy) imaging at day 7 reflected this quantitative data showing a reduction in the number of the large osteoclasts (Fig. 2e). To study osteoclast activity, standard 24 well plates were replaced with 24 well Osteo Assay surface plates and percentage area resorbed measured after 7 days of culture. With nanovibrational stimulation, significantly less resorption was observed (Fig. $2 \mathrm{f}$ ). The reduction in resorption was similar to that of the osteoclast cell count (approximately $30 \%$ for both resorption and cell numbers) which suggests the number of osteoclasts rather than their ability to resorb has been primarily affected. This is important, given the requirement of functional osteoclasts for normal bone remodelling and homeostasis ${ }^{8}$.

Looking at 2D qPCR transcriptional data for the $\mathrm{CD} 14^{+}$cultures, genes involved is osteoclast formationTRAP and OSCAR (osteoclast-associated receptor) ${ }^{26}$ had a slight trend towards increased up-regulation with nanovibrational stimulation at day 2 and 3 (Fig. 2g). However, these changes did not reach statistical significance; an increase in cathepsin $\mathrm{K}$ at day 1 in the nanovibrational group was the only significant difference observed. Further, inflammatory transcripts IL-6 (interleukin 6), TNFa (tumour necrosis factor alpha) ${ }^{27}$ and NFATc1 (nuclear factor of activated T-cells, cytoplasmic 1) did not change between control and nanovibrated cultures (Fig. 2g). By day 7, all transcripts, apart from cathepsin K, were strongly repressed (Fig. 2g) across both control and nanovibrated cultures. It is perhaps to be expected that no change in expression of the RANKL decoy receptor and negative mediator of osteoclast formation, osteoprotegrin $(\mathrm{OPG})^{13}$ was noted at any time point, as there were no osteoblast forming cells in culture. However, we note that OPG can be expressed in osteoclast monocultures 

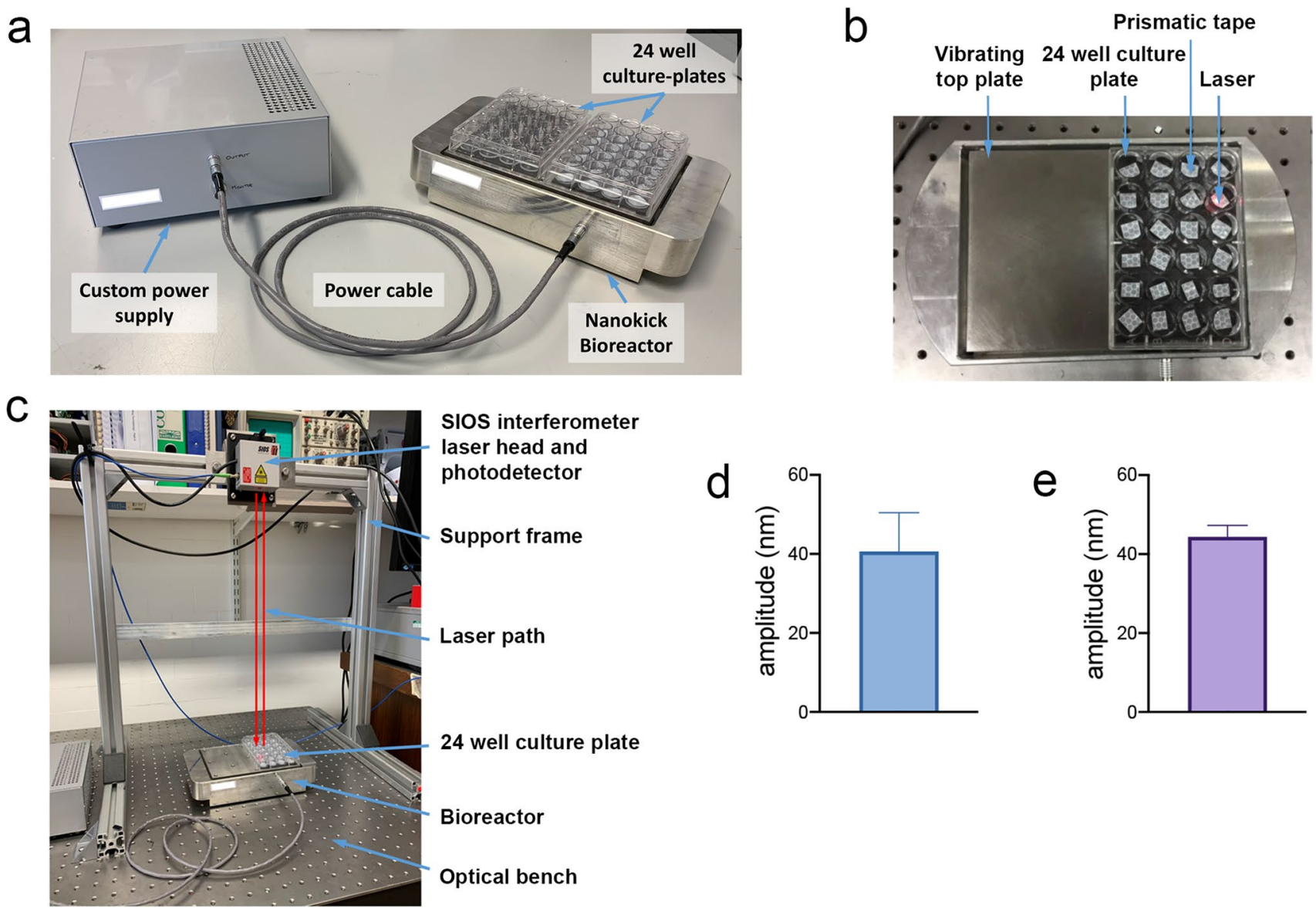

SIOS interferometer

laser head and

photodetector

Support frame
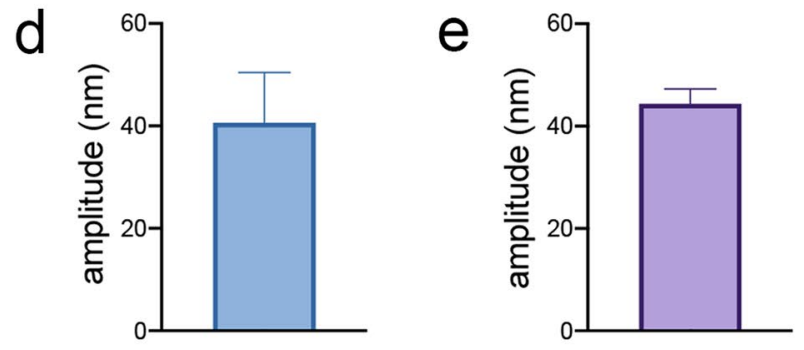

24 well culture plate

Bioreactor

Optical bench

Figure 1. Nanovibrational stimulation setup and measurement. (a) Nanovibrational bioreactor and power supply. (b) 24 well cell culture plate attached to the bioreactor using a magnetic sheet. Reflective prismatic tape is placed in the wells (or on top of gels) so that the interferometer laser is reflected back to the detector. (c) Interferometer measuring bioreactor vibrations in the 24 well plate. (d) 2D nanovibrations in two 24 well plates. No cells were present during measurement. Measurements were taken in triplicate from each well, giving an average displacement of $40.6 \mathrm{~nm}$ at $1000 \mathrm{~Hz}$ frequency. (e) 3D (collagen gel) nanovibrations in a 24 well plate giving an average displacement of $44.4 \mathrm{~nm}$ at $1000 \mathrm{~Hz}$ frequency.

as part of a self-regulatory mechanism, potentially inducing apoptosis ${ }^{28}$. Looking at protein expression of inflammatory mediators IL- 6 and TNFa, and osteoclast inhibitor OPG by ELISA (enzyme-linked immunosorbent assay), very little change was seen after 3 days of culture; only expression of IL- 6 was seen to be reduced by nanovibrational culture (Fig. 2h).

The qPCR data suggests that the monocytes follow normal gene expression patterns for both control and nanovibrated cultures and that there is no remarkable difference in expression. In order to assess if other pathways could be inferred, we used untargeted metabolomics after 3 days of nanovibrational culture. Ingenuity pathway analysis, a well curated literature-based pathway building software, was used for bioinformatics. Using molecular pathway prediction, the most changed network was around NFKB (nuclear factor kappa-light-chain-enhancer of activated B cells), predicting down-regulation with nanovibrational stimulation (Supplementary Fig. 3). NFkB is known to activate osteoclast differentiation factors c-Fos and NFATc1 (Nuclear factor of activated T-cells, cytoplasmic 1) in response to RANKL and is required for terminal osteoclast differentiation. While this seems a sensible target, we note that no activation of NFATc1 in either control of nanovibrated samples was noted in Fig. $2 \mathrm{~g}$ and so further analysis is required ${ }^{29,30}$.

Together these data shows an inhibitory effect of nanovibration on osteoclast differentiation and function. These results are in agreement with previous studies that have shown slowed bone resorption with $45 \mathrm{~Hz}$ whole body vibration ${ }^{31}$ and that low-intensity pulsed ultrasound at $1.5 \mathrm{MHz}$ inhibits RANKL induced osteoclast formation $^{32}$. However, looking at standard markers of osteoclast phenotype progression, only subtle changes were noted.

Up-regulation of osteogenesis and inhibition of osteoclastogenesis in BMSC/BMHC co-culture. For the co-culture, wells of 24 well culture plates were flooded with $3 \times 10^{4}$ cells $/ \mathrm{ml}$ of BMSCs in $1 \mathrm{ml}$ culture media. BMSCs were isolated from human bone marrow following Ficoll gradient selection and then culturing the cells on tissue culture plates for 3 days. The adherent cells comprise the whole stromal fraction containing osteoprogenitor and mesenchymal stem cells. Concurrently, the non-adherent BMHC culture was 
a

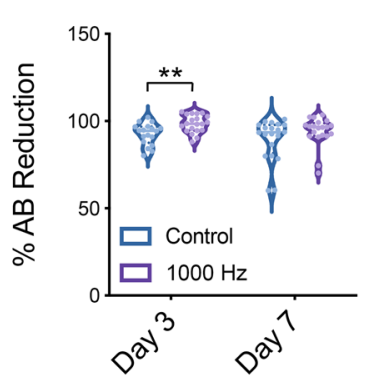

b

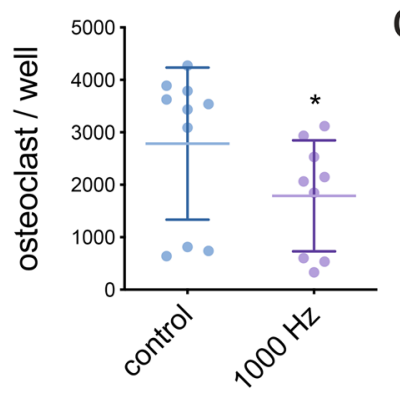

䍃 100

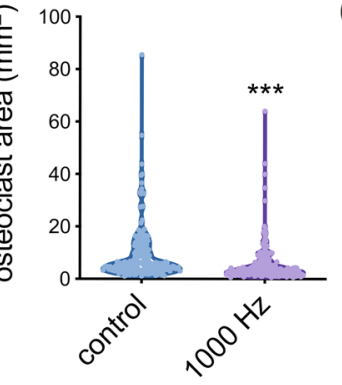

$1000 \mathrm{~Hz}$
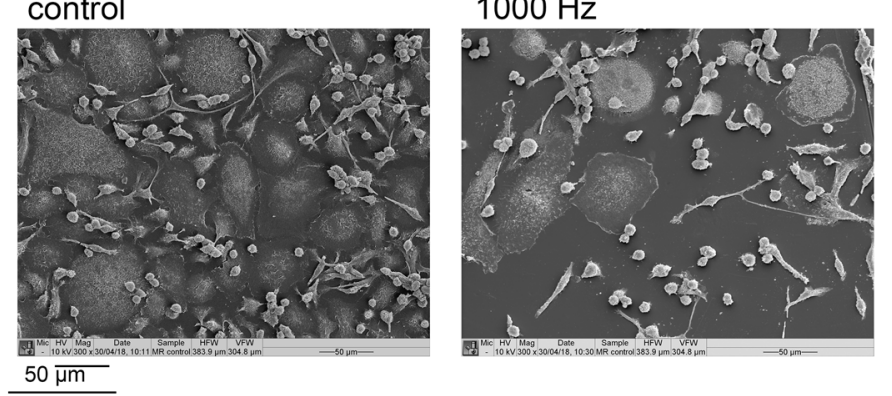

$f$

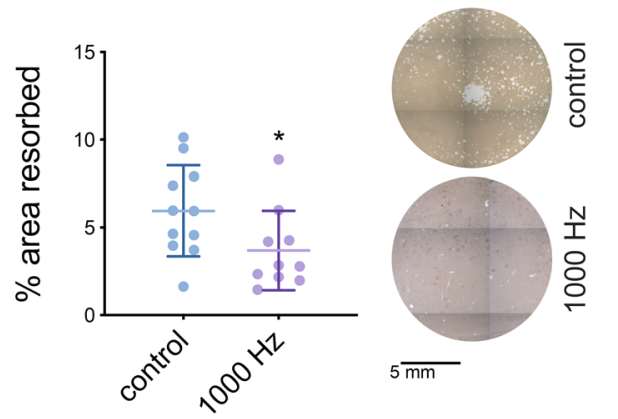

\begin{tabular}{|c|c|c|c|c|c|c|c|c|}
\hline \multirow{2}{*}{$9_{2^{-\triangle \Delta C T}}$} & \multicolumn{2}{|c|}{ day 1} & \multicolumn{2}{|c|}{ day 2} & \multicolumn{2}{|c|}{ day 3} & \multicolumn{2}{|c|}{ day 7} \\
\hline & control & $1000^{\mathrm{Hz}}$ & control & $1000^{H 2}$ & $\mathrm{con}^{\mathrm{ntrol}}$ & $1000^{\mathrm{Hz}}$ & control & $1000^{H 2}$ \\
\hline TRAP & 2.99 & 3.43 & 0.83 & 1.01 & 1.41 & 1.67 & 0.32 & 0.3 \\
\hline OSCAR & 2.28 & 1.5 & 4.05 & 7.07 & 1.49 & 1.61 & 0.43 & 0.26 \\
\hline Cathepsin K & 1.61 & * 2.94 & 1.23 & 1 & 12.5 & 9.5 & 13.6 & 13.63 \\
\hline RANK & 1.44 & 1.31 & 1.43 & 2.94 & 1.05 & 1.27 & 1.38 & 3.52 \\
\hline CSF-1 & 1.26 & 1.99 & 1.01 & 0.94 & 1.01 & 1.41 & 1.11 & 1.29 \\
\hline NFATC1 & 1.36 & 6.9 & 1 & 0.7 & 1.04 & 1.06 & 1.09 & 1.85 \\
\hline IL6 & 0.62 & 0.69 & 1.09 & 0.95 & 0.85 & 0.69 & 0.03 & 0.1 \\
\hline TNF $\alpha$ & 1.24 & 1.31 & 0.89 & 1.14 & 0.82 & 0.51 & 0.26 & 0.09 \\
\hline
\end{tabular}

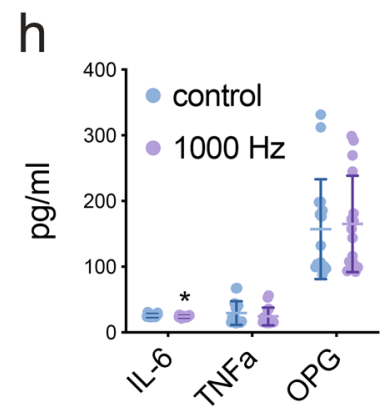

Figure 2. Osteoclast response to nanovibrational stimulation in 2D. (a) No detrimental effect on CD14 ${ }^{+}$ cell viability was seen, as measured by Alamar blue (violin plots of individual data points, $n=(d=3, r=3)$, statistics by t-test where $\left.{ }^{*} \mathrm{p}<0.05\right)$. However, $(\mathbf{b})$ following 7 days of nanovibrational stimulation numbers of fused, multinucleate osteoclasts observed by TRAP staining were reduced (mean \pm SD of individual data points, $n=(d=3, r=3)$, statistics by t-test where $\left.{ }^{\star} p<0.05\right)$. Similarly, the mean osteoclast area (c) was also reduced with nanovibrational stimulation (violin plots of individual data points, $n=(d=3, r=3)$, statistics by t-test where ${ }^{* *} \mathrm{p}<0.001$ ) after 7 days of culture; (d) typical TRAP staining of both control and $1000 \mathrm{~Hz}$ stimulated samples imaged at $10 \times$ magnification. (e) SEM images at day 7 showing less osteoclasts were present following nanovibrational stimulation. (f) Resorption assay at day 7 showing less osteoclast activity following nanovibrational stimulation (mean \pm SD of individual data points, $\mathrm{n}=(\mathrm{d}=3, \mathrm{r}=3$ ), statistics by $\mathrm{t}$-test where $\left.{ }^{*} \mathrm{p}<0.05\right)$. (g) qPCR for nanovibrated vs control CD14 ${ }^{+}$cells for transcripts related to osteoclastogenesis and inflammation. A trend towards repression of these genes in the nanovibrated cultures was observed $\left(\mathrm{n}=(\mathrm{d}=1-3, \mathrm{r}=3)\right.$, statistics by t-test where $\left.{ }^{\star} \mathrm{p}<0.05\right)$; full qPCR data is presented in Supplementary Fig. 2. (h) At the protein level, IL- 6 was seen to be repressed (mean \pm SD of individual data points, $n=(d=3, r=4)$, statistics by $\mathrm{t}$-test where $\left.{ }^{\star} \mathrm{p}<0.05\right)$. Together, the data indicates a reduction in osteoclast forming activity of CD $14^{+}$blood mononuclear cells with nanovibrational stimulation.

maintained in T75 flasks until cells, presumed to be monocytes, started to adhere. At 7 days of BMSC culture, these osteoclast progenitor cells were added in to the 24 well plate at $1.2 \times 10^{5} \mathrm{cells} / \mathrm{ml}$ during media change with $1 \mathrm{ml}$ of culture media. The addition of the osteoclast progenitor cells was considered day 0 of the co-culture. No supplement of MCSF or RANKL was used as the BMSCs stimulate osteoclast fusion from macrophages in this culture system. Longer time points (i.e., days 7, 14, 21 and 28) than those used in the CD14 ${ }^{+}$culture were used to account for the lack of supplementary cytokine (and to allow sufficient time to observe osteogenesis). 
Looking at viability at days 7, 14 and 21 using Alamar blue, comparable reduction levels were seen for control and nanovibrated cultures (Fig. 3a). At 28 days of co-culture, TRAP staining was used to identify osteoclasts and it was seen that number of osteoclasts and size of osteoclasts (as a measure of fusion) decreased with nanovibrational culture (Fig. 3b-d shows typical TRAP images for control (left) and $1000 \mathrm{~Hz}$ stimulated (right) cultures). Furthermore, at day 28 of co-culture, actin cytoskeleton/DAPI and SEM images were taken. In line with quantitative data on size (Fig. 3b,c), osteoclasts (denoted by multinuclei and typical actin ring) tended to have fewer nuclei per cell (Fig. 3e) and tended to be smaller (Fig. 3f). SEM images clearly showed the macrophage, osteoclast and BMSC co-culture (Fig. 3f).

Considering osteogenesis of the BMSCs in co-culture, von Kossa stain for mineralization was employed at day 28 of co-culture. First looking at 2D BMSC monoculture, enhanced mineralization was seen as expected (Fig. 3 g) ${ }^{20}$. For the $2 \mathrm{D}$ co-culture, mineralization was seen to be highly significantly enhanced (Fig. $3 \mathrm{~g}$ ). Finally, for the $3 \mathrm{D}$ co-culture within collagen gels BMSC mineralization was seen to be significantly increased. This shows, for the first time, that nanovibrational osteogenesis is maintained in co-culture conditions.

Next, qPCR was used to measure transcripts related to the BMHC (TRAP, OSCAR, Cathepsin K, IL6, TNFa and OPG) and BMSC (RANKL, M-CSF, alkaline phosphatase (ALP), osteopontin (OPN), osterix and the mechanosensitive ion channel piezo 1 , all of which relate to the osteoblast phenotype) populations in $2 \mathrm{D}$ and $3 \mathrm{D}$ co-cultures. For both $2 \mathrm{D}$ and $3 \mathrm{D}$ co-cultures, there was a trend, mainly in the osteoclast maturity transcripts, that with nanovibration, transcripts were up-regulated at day 7 compared to control cultures but then tended to become down-regulated at days 14, 21 and 28 (Fig. 3h). In 2D, this included significant reductions in IL-6 at day 14 and OSCAR at day 21 in the $2 \mathrm{D}$ culture. Considering osteogenic transcripts, a non-significant trend of increased osteogenic transcript expression (ALP and OPN) was noted in 2D. In 3D, the osteogenic pattern was more apparent with significant upregulations in OPN transcript expression, as well as increased trends of expression for ALP and for piezo 1, a mechanosensitive ion channel implicated in osteogenesis ${ }^{20,33}$. This data supports osteogenesis occurring even in co-culture where RNA from both BMSCs and BMHCs was isolated.

Lipid expression, involved in energy pathways, are regularly cited as changing with physical stimuli ${ }^{34-36}$. Thus, untargeted metabolomic analysis was employed to study fold change to unstimulated control between 2 and 3D nanovibrational co-cultures. At both 14 and 21 days of co-culture, it was seen that lipid-based pathways were differentially regulated in nanovibrated cultures (2D and 3D) compared to control cultures based on patterns of lipid abundance (Fig. 3i). This difference was most apparent at the earlier time point of culture (day 14). This suggests, potentially, that cell growth and differentiation is more energetically demanding in $3 \mathrm{D}$ culture compared to 2D culture. Further, it is known that depletions of steroids (e.g. dexamethasone) and cholesterols (e.g. cholesterol sulphate) are important in osteogenesis and this was observed in nanovibrated cultures compared to controls (Fig. 3i) ${ }^{37-39}$.

Further pathway analysis of untargeted metabolomics data found that metabolites feeding into protein kinase $\mathrm{B}$ (Akt) could be found to be differentially regulated compared to controls at both days 14 and 21 of co-culture for $2 \mathrm{D}$ and $3 \mathrm{D}$ nanostimulation, with Akt expression predicted to be up-regulated at day 14 and down-regulated at day 21 (Supplementary Fig. 6). Akt is known to be important in osteogenesis and osteoclast formation ${ }^{40}$.

\section{Discussion}

We report on a mechanical bioreactor that provides nanoscale stimulation to osteoblastic cells while reducing osteoclast formation in appropriate co-culture conditions. Such a methodology could find use in several ways: as a tissue engineering approach, as a direct therapy and as a drug discovery platform.

Bone is the second most commonly transplanted tissue after blood ${ }^{41}$. The current gold-standard is autologous bone graft (i.e., harvested from the same individual) due to its osteogenic, osteoconductive and osteoinductive properties $^{41}$. However, allograft-in such forms as demineralised bone matrix, morcellised cancellous chips or whole bone segments-are also frequently utilised ${ }^{41}$. There are well-evidenced disadvantages to the current graft options, including donor site pain and complications ${ }^{42,43}$, and off-target effects in the case of BMP- $2^{44,45}$. Tissue engineering approaches, such as drug-free 3D bone differentiation, as we demonstrate here, represent an attractive option to overcome the aforementioned challenges and as such have been an area of significant interest in the scientific community ${ }^{46}$.

As well as stimulating osteogenesis of BMSCs, we demonstrate, for the first time, reduction of osteoclast formation and activity in $2 \mathrm{D}$ and $3 \mathrm{D}$ co-cultures. This is important as the interplay between osteoblasts and osteoclasts is crucial to normal bone development, homeostasis and remodelling after injury. Any imbalance in this dynamic, particularly with regard to osteoclast function, can lead to pathological conditions, such as osteoporosis $^{8,47}$ and osteopetrosis ${ }^{48,49}$. With the ageing population, in whom degenerative and pathological conditions such as osteoporosis are more prolific, the demand for innovation has never been higher ${ }^{46,50,51}$. As discussed, application of whole body vibration ${ }^{31}$ and low-intensity pulsed ultrasound inhibit osteoclastogenesis $\mathrm{s}^{32}$. There are also indications that these vibration approaches may aid bone regeneration ${ }^{52-55}$. However, it is difficult to determine from in vivo studies whether it is muscle tone, bone regeneration or osteoclast formation that is altered, and in vitro studies that look at only one cell type in isolation are limited as a consequence ${ }^{52-55}$. Our approach allows us to understand that both bone formation and osteoclast fusion and activity are effected at the intermediate, $1000 \mathrm{~Hz}$, frequency. This allows us to envisage a direct application of nanovibration, as with whole body vibration ${ }^{56}$ and low-intensity pulsed ultrasound ${ }^{57}$, after further in vivo evaluation using technologies similar to bone conduction headphones $(1000 \mathrm{~Hz} \text { is an audible signal })^{58}$. Our human, 3D model of bone homeostasis where osteogenesis is enhanced and osteoclastogenesis reduced, and where potential pathways can be identified, could also be useful in researching new drugs for conditions such as osteoporosis. Further validation of the metabolomic findings could strengthen the use of this model. 
a
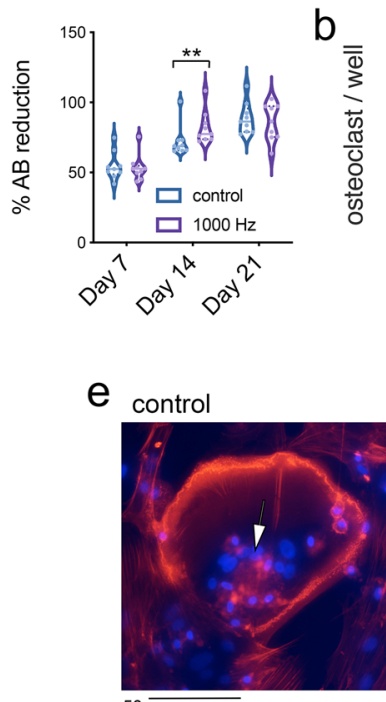

$1000 \mathrm{~Hz}$

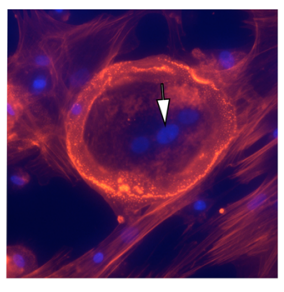

$50 \overline{\mu \mathrm{m}}$

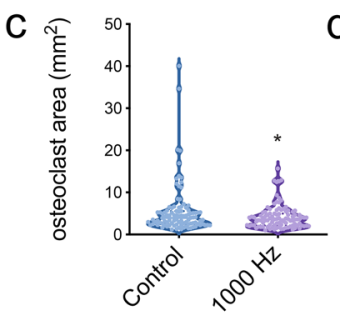

$0^{x^{x i v e}}$

f control

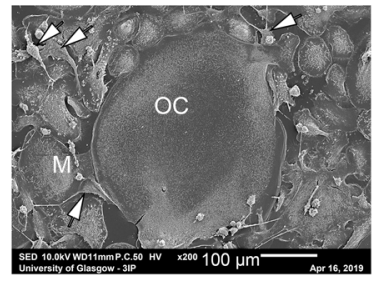

d control $\because 31000 \mathrm{~Hz}$

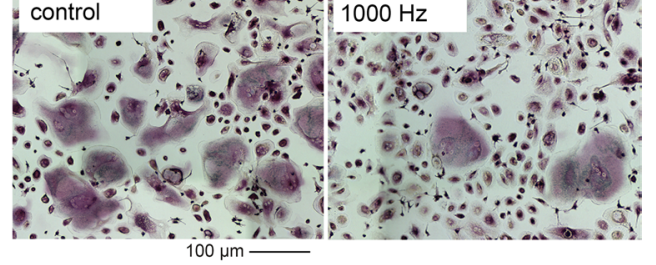

g

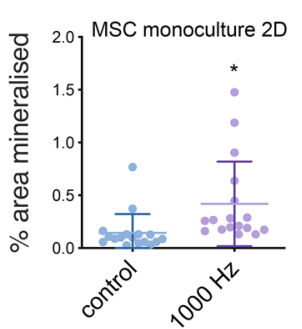

$5 \mathrm{~mm}$
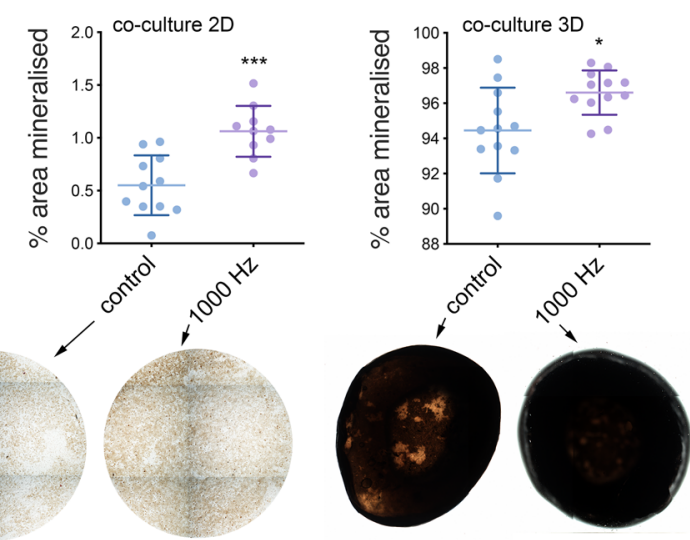

h

day 7

day 14

day 21

day 28

\begin{tabular}{|c|c|c|c|c|c|c|c|c|}
\hline & & & & & & & & \\
\hline $2^{-\triangle \Lambda C T}$ & $\mathrm{con}^{0+\mathrm{tr}}$ & 1000 & control & $1000^{r}$ & control & 1000 & control & $1000^{\mathrm{Hz}}$ \\
\hline TRAP & 1.82 & 2.02 & 1.13 & 1.07 & 1.32 & 0.74 & 1.88 & 0.37 \\
\hline OSCAR & 1.03 & 1.94 & 1.02 & 1.01 & 1.12 & * 0.73 & 1.74 & 0.55 \\
\hline athepsin $\mathrm{K}$ & 1.04 & 2.18 & 1.05 & 0.87 & 1.09 & 0.77 & 1.22 & 1.25 \\
\hline RANKL & 1.15 & 1.45 & 1.17 & 0.73 & 1.75 & 2.15 & 1.29 & 1.24 \\
\hline M-CSF & 1.05 & 2.17 & 1.09 & 0.82 & 1.17 & 1.18 & 1.28 & 0.63 \\
\hline IL-6 & 1.01 & 1.02 & 1.06 & $* * 0.6$ & 1.11 & 1.18 & 1.09 & 0.6 \\
\hline TNF $\alpha$ & 1.02 & 2.14 & 1.05 & 1.08 & 1.09 & 0.88 & 1.11 & 0.79 \\
\hline OPG & 1.05 & 3.17 & 1.01 & 2.7 & 1.05 & 0.98 & 1.23 & 0.55 \\
\hline ALP & 1.02 & 3.01 & 1.07 & 1.31 & 1.22 & 0.75 & 1.19 & 0.56 \\
\hline OPN & 1.2 & 1.85 & 1.09 & 0.77 & 1.22 & 1.25 & 1.43 & 1.54 \\
\hline
\end{tabular}

\begin{tabular}{r|r} 
TRAP & 1.04 \\
OSCAR & 1.16 \\
Cathepsin K & 1.03 \\
RANKL & 1.1 \\
M-CSF & 1.04 \\
IL-6 & 1.01 \\
TNF $\alpha$ & 1.03 \\
OPG & 1.09 \\
ALP & 1.04 \\
OPN & 1.24 \\
Osterix & \\
\hline Piezo &
\end{tabular}

Piezo 1

$\begin{array}{rrrr}1.04 & 1.74 & 1.67 & 1.26 \\ 1.16 & 0.94 & 2.15 & 1.11 \\ 1.03 & * 3.12 & 1.23 & 2.59 \\ 1.1 & 1.22 & 1.72 & 0.89 \\ 1.04 & 0.88 & 1.89 & 0.81 \\ 1.01 & 2.31 & 1.7 & 0.91 \\ 1.03 & 1.18 & 1.76 & 1.03 \\ 1.09 & 1.2 & 1.84 & 1.42 \\ 1.04 & 1.37 & 1.62 & 1.08 \\ 1.24 & * * * 8.78 & 1.89 & 4.63 \\ 0 & 0 & 0 & 0 \\ 0 & 0 & 1 & 3.8\end{array}$

$2 \mathrm{D}$

$\begin{array}{rr}1.2 & 1.56 \\ 1.2 & 1.67 \\ 1.41 & 1.7 \\ 1.42 & 1.05 \\ 1.27 & 0.97 \\ 1.38 & 0.89 \\ 1.41 & 0.95 \\ 1.37 & 1.01 \\ 1.49 & 0.75 \\ 1.6 & 3.69 \\ 1 & 1.17 \\ 1.1 & 1.79\end{array}$

$\left.\begin{array}{rr}1.07 & 2.53 \\ 1.22 & 1.08 \\ 1.06 & 1.05 \\ 1.13 & 3.33 \\ 1.07 & 1.87 \\ 1.12 & 2.7 \\ 1.06 & 1.85 \\ 1.07 & 2.68 \\ 1.14 & 3.56 \\ 1.05 & 1.32 \\ 1 & 2.62 \\ 1.06 & 2.19\end{array}\right] 3 \mathrm{D}$

$1000 \mathrm{~Hz}$

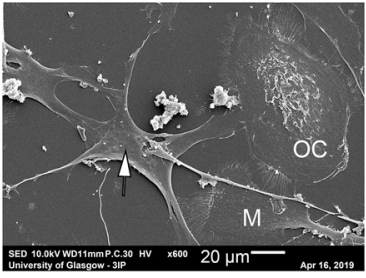

จิ ले

Activation z-score $\frac{\pi}{0}$ 完 $2.592 \quad$ サ Concentration of acylglyc Transport of eicosano Accumulation of lipid Concentration of triacylgl... Uptake of lipid

Accumulation of acylglyce. Storage of triacylglycerol Quantity of steroid Transport of fatty acid Binding of phospholipid Synthesis of fatty acid Concentration of stero Accumulation of triacylgly. Synthesis of prostagland in * Concentration of choleste.

Fatty acid metabolism

Uptake of fatty acid Hydrolysis of lipid Hydrolysis of lipid

Accumulation of sphingoli... Lipolysis

Transport of phos pholipid Concentration of acyl-co Synthesis of acylglycerol Accumulation of ceramide Conversion of fatty acid

Quantity of polyunsaturat. Hydrolysis of glycosphing Release of lipid Binding of lipid Concentration of phosph. Excretion of lipid Concentration of malonyl... Release of fatty acid Transport of lipid Synthesis of eicosanoid Secretion of steroid Efflux of phospholipid Concentration of eicosan Secretion of lipid Concentration of prostagl... Hydrolysis of sphingolipid Flux of lipid

Synthesis of triacylglycerol Esterification of lipid Synthesis of lipid Concentration of lipid Lipolysis of cells

Hydrolysis of phospholipid Synthesis of ceramide
ते ले Activation z-score 永 -3.179 2.740 స Lipolysis Concentration of acylglyc. * Quantity of steroi Hydrolysis of lipid Concentration of lipid Concentration of sterol Accumulation of acylglyce. Concentration of triacylgl... Hydrolysis of sphingolipid Efflux of phospholipid * Concentration of choleste. Conversion of lipid Transport of phos pholipid Concentration of fatty acid Transport of fatty acid Accumulation of sphingoli. Synthesis of acylglycerol Metabolism of sphingolipid Hydrolysis of glycosphing. Accumulation of lipid

Accumulation of triacylgly... Accumulation of ceramide Conversion of fatty acid Storage of triacylglycerol Concentration of acyl-coe. Synthes is of lipid

Synthesis of sphingolipid Synthesis of ceramide

Hydrolysis of glucosylcer... Metabolism of membrane Binding of phospholipid Transport of lipid Fatty acid metabolism Concentration of prostagl. Transport of eicosanoid. Concentration of phosph. Quantity of polyunsaturat. Quantity of polyunsatu
Synthesis of fatty acid Synthes is of fatty acid
Synthesis of triacylglycero 
«Figure 3. BMSC and BMHC co-culture in 2D and 3D. (a) No detrimental effect on cell viability, as measured by Alamar blue, was seen (violin plots of individual data points, $\mathrm{n}=(\mathrm{d}=3, \mathrm{r}=3)$, statistics by $\mathrm{t}$-test where $\left.{ }^{* *} \mathrm{p}<0.01\right)$. Using TRAP stain to identify osteoclasts after 28 days of co-culture, $(\mathbf{b})$ the number of osteoclasts was seen to reduce (mean \pm SD of individual data points, $n=(d=3, r=3)$, statistics by t-test where ${ }^{\star *} \mathrm{p}<0.01$ ) and (c) area of osteoclasts was decreased with nanovibrational stimulation (violin plots of individual data points, $\mathrm{n}=(\mathrm{d}=3, \mathrm{r}=3)$, statistics by $\mathrm{t}$-test where $\left.{ }^{\star} \mathrm{p}<0.05\right)$. (d) Typical TRAP stain of both control and $1000 \mathrm{~Hz}$ stimulated samples imaged at $10 \times$ magnification. (e) Actin/DAPI immunofluorescence after 28 days of culture showed that osteoclast cells identified by multiple nuclei and by actin rings tended to have fewer nuclei following nanovibrational stimulation. Arrows indicate the multiple nuclei of each cell. (f) SEM images after 28 days of culture showed that while many osteoclasts could be seen in control co-cultures, fewer were observed, along with better spread BMSCs, following nanovibrational stimulation. Arrows indicate BMSCs; $\mathrm{M}=$ macrophage; $\mathrm{OC}=$ osteoclast. $(\mathrm{g})$ Looking at osteogenesis after 28 days of culture using von Kossa staining in 2D BMSC monoculture, 2D co-culture and 3D co-culture, osteogenesis was enhanced in all conditions with nanovibrational stimulation (mean \pm SD of individual data points, $\mathrm{n}=(\mathrm{d}=3, \mathrm{r}=4-5)$, statistics by $\mathrm{t}$-test where $\left.{ }^{*} \mathrm{p}<0.05,{ }^{* *} \mathrm{p}<0.001\right)$; typical von-Kossa images from the co-cultures are shown below their corresponding graphs $(2 \mathrm{D}=$ left, $3 \mathrm{D}=$ right). (h) qPCR for nanovibrated vs control $2 \mathrm{D}$ (top) and 3D (bottom) co-cultures for transcripts related to osteoclastogenesis, inflammation and osteogenesis showing a trend towards initial activation and then repression of osteoclast-related genes and activation of osteoblast related genes for nanovibrated cultures $\left(\mathrm{n}=(\mathrm{d}=1-4, \mathrm{r}=3-4)\right.$, statistics by $\mathrm{t}$-test where ${ }^{\star} \mathrm{p}<0.05,{ }^{* *} \mathrm{p}<0.01$ and $\left.{ }^{\star * *} \mathrm{p}<0.001\right)$. Together, the data indicates reduction in osteoclast forming activity and increase in osteoblast forming activity of the co-cultures in both 2D and 3D with nanovibrational stimulation. Full qPCR data is presented in Supplementary Figs. 4 and 5. (i) Untargeted metabolomic analysis for 2D and 3D co-culture. Lipid-based pathways were upregulated, particularly at day 14 in the 3D culture; steroid and cholesterol pathways are indicated by ${ }^{\star}$. This suggests that cell growth and differentiation is more energetically demanding in $3 \mathrm{D}$ culture compared to $2 \mathrm{D}$ culture $(\mathrm{n}=3)$.

\section{Conclusion}

$1000 \mathrm{~Hz}$ nanovibrational stimulation reduces osteoclast formation and activity in monocultures. In primary 2D and 3D human osteoprogenitor/osteoclast progenitor co-cultures, the $1000 \mathrm{~Hz}$ stimulation leads to enhanced osteogenesis and reduced osteoclast formation. This provides a model of bone remodelling where bone formation is dominant to loss and use of untargeted metabolomics allows identification of controlling pathways.

\section{Materials and methods}

This work has been approved by the NHS research Scotland (NRS) Greater Glasgow and Clyde Bio-repository ethics committee. All methods were carried out in accordance with relevant guidelines and regulations. Informed consent was obtained from all subjects who donated tissue samples.

$\mathrm{CD}_{14}{ }^{+}$culture. Buffy coats were received from the Scottish National Blood Transfusion Service. Magnetic selection was performed using EasySep Human CD14 positive selection kit II (product no. 17858, Stemcell Technologies, Cambridge, UK. The cells were re-suspended at $1 \times 10^{6}$ cells $/ \mathrm{ml}$ in $\alpha$-MEM (alpha minimum essential media) supplemented with $10 \%$ FBS, $0.02 \mathrm{mM}$ L-glutamine, $10 \mathrm{U} / \mathrm{ml}$ Penicillin, and $0.1 \mu \mathrm{g} / \mathrm{ml}$ Streptomycin (all components from Sigma-Aldrich, Dorset, UK). Following suspension at $1 \times 10^{6}$ cells $/ \mathrm{ml}, 25 \mathrm{ng} / \mathrm{ml}$ of recombinant human M-CSF (product no. 300-25, Peprotech, London, UK) was added. Cells were plated in 24 well plates and incubated overnight at $37^{\circ} \mathrm{C}$ and $5 \% \mathrm{CO}_{2}$. After approximately $18 \mathrm{~h}$ incubation $25 \mathrm{ng} / \mathrm{ml}$ of human RANKL (product no. 310-01, Peprotech, London, UK) was added to a proportion of the wells. Those wells with M-CSF and no RANKL were used as a negative control of osteoclastogenesis.

Co-culture. Human bone marrow was aspirated from patients undergoing elective hip and knee arthroplasty. The bone marrow aspirate was washed and centrifuged to produce a cell pellet. The cell pellet was resuspended and overlaid on a Ficoll-Paque 1.073 (No. 17-5446-52, Sigma-Aldrich, Dorset, UK) gradient and the subsequent mononuclear interface layer aspirated and resuspended in mDMEM ((dulbecco's Modified Eagle Medium) (Sigma-Aldrich, Dorset, UK), 10\% FBS (Sigma-Aldrich, Dorset, UK), 1\% Sodium Pyruvate (11 $\mathrm{mg} \mathrm{ml}^{-1}$, Sigma-Aldrich, Dorset, UK), 1\% Gibco MEM NEAA (non-essential amino acids, Thermo Fisher Scientific, Loughborough, UK), $2 \%$ antibiotics $\left(6.74 \mathrm{U} \mathrm{ml}^{-1}\right.$ penicillin-streptomycin (Sigma-Aldrich, Dorset, $\mathrm{UK}$ ) and $0.2 \mu \mathrm{g} \mathrm{m}^{-1}$ fungizone (Sigma-Aldrich, Dorset, UK)). The cells were then plated at a density of $1 \times 10^{6}$ in a $75 \mathrm{~cm}^{2}$ vented cell culture flask and incubated at $37^{\circ} \mathrm{C}$ with $5 \%$ humidified $\mathrm{CO}_{2}$. At day 3 non-adherent cells were removed and cultured separately. This non-adherent fraction contained mainly bone marrow haematopoietic cells (BMHC), macrophages and osteoclast precursors. The remaining adherent cells were assumed to be mesenchymal stromal cells (MSCs), osteoprogenitors, osteoblasts and osteocytes and were cultured until an approximately $80 \%$ confluent layer was obtained. The adherent cells were then detached and resuspended in $\mathrm{mDMEM}$ to a concentration of $3 \times 10^{4}$ cells $/ \mathrm{ml}$. $1 \mathrm{ml}$ of cell suspension/well was pipetted onto a 24 well plate. At day $7,1 \mathrm{ml}$ of BMHC suspension/well was added at a concentration of $1.2 \times 10^{5} / \mathrm{ml}$.

3D cell culture technique. Cells were isolated as discussed. Type 1 rat collagen gel was then prepared by adding sodium hydroxide $(\mathrm{NaOH}), 10 \%$ FBS and $10 \times$ DMEM. Cells were added to the gel and further $\mathrm{NaOH}$ was added drop-wise while agitating the mix until the colour changed from yellow to pink, indicating the correct 
$\mathrm{pH}$ had been achieved. $1 \mathrm{ml}$ of gel/well was pipetted on to a 24 well plate. $1 \mathrm{ml}$ of media \pm growth factors was added on top of the gels after $30 \mathrm{~min}$.

TRAP staining. Cells were fixed after 7 days for the $\mathrm{CD} 14^{+}$culture and 28 days for the co-culture. TRAP (No. 387A, Sigma-Aldrich, Dorset, UK) was used as per manufacturer's instructions. Samples were then washed and air dried. Digital images of the entire wells were acquired. Osteoclasts were identified as TRAP positive cells with $\geq 3$ nuclei and quantified by manual counting of both number and area.

Immunostaining. Co-culture samples were cultured for 28 days. Medium was removed and cells were fixed with 3.7\% methanol-free formaldehyde (Sigma-Aldrich, Dorset, UK) for $10 \mathrm{~min}$ at room temperature. Samples were then washed twice with $1 \times$ PBS (phosphate buffer saline) and permeabilised with $0.1 \%$ Triton $\mathrm{x}-100$. Rhodamine-conjugated phalloidin (No. R415, Thermo Fisher Scientific, Loughborough, UK) was added for an hour in the dark $(1: 300$ in $1 \%$ BSA (bovine serum albumin) $/ 1 \times$ PBS). Samples were washed before adding DAPI and viewing the samples with a fluorescent microscope to visualise the actin rings and nuclei using an EVOS ${ }^{\circ}$ FL Auto 2 Cell Imaging System.

Von Kossa staining. Samples were cultured for 28 days then fixed with $4 \%$ formaldehyde solution. $5 \%$ silver nitrate was added before exposing to UV light for $30 \mathrm{~min}$. Samples were washed, then 5\% sodium thiosulphate added for $10 \mathrm{~min}$. A further wash was performed before counterstaining with $0.1 \%$ nuclear fast red. A final wash with deionized water was performed then a further rinse with $70 \%$ ethanol. Images of the entire well were obtained and the percentage surface area mineralised calculated.

Scanning electron microscopy. Cells were fixed with buffered glutaraldehyde fixative for $1 \mathrm{~h}$ at $4{ }^{\circ} \mathrm{C}$ then rinsed with $0.1 \mathrm{M}$ sodium cacodylate. Postfix in $1 \%$ osmium tetroxide for $1 \mathrm{~h}$ at room temperature was then performed. A dehydration process was carried out using an ethanol series. Hexamethyl-disilazane was then applied to samples and left overnight for drying. An $18 \mathrm{~nm}$ gold palladium coating was overlaid on the sample surface using a Polaron SC515 SEM Coating System. Finally, samples were attached to aluminium stubs and analysed on a Carl Zeiss Sigma variable-pressure analytical SEM ${ }^{20}$.

Alamar Blue assay. Cell viability was assessed at defined time points using an Alamar Blue resazurin (No. DAL1025, Thermo Fisher Scientific, Loughborough, UK) assay according to the manufacturer's instructions. A $10 \%$ solution was produced. Media was removed from the wells and $500 \mu \mathrm{l}$ of $10 \%$ Alamar Blue added. Both the stimulated and control samples were then incubated at $37^{\circ} \mathrm{C}$ for $4 \mathrm{~h}$ in the dark. $150 \mu \mathrm{l} /$ well of $10 \%$ Alamar Blue was then transferred in triplicate to a 96 well plate. The remainder of $10 \%$ Alamar Blue was discarded before washing with HEPES saline and adding fresh media. The absorbance was measured at $570 \mathrm{~nm}$ and $600 \mathrm{~nm}$ using a Multiskan FC Microplate Photometer ${ }^{20}$. The percentage of Alamar Blue reduction was calculated using the formula:

$$
\% \text { reduction of Alamar Blue }=((\mathrm{O} 2 \times \mathrm{A} 1)-(\mathrm{O} 1 \times \mathrm{A} 2) /(\mathrm{R} 1 \times \mathrm{N} 2)-(\mathrm{R} 2 \times \mathrm{N} 1)) \times 100
$$

$\mathrm{O} 1$ and $\mathrm{O} 2=$ the molar extinction coefficients of oxidised Alamar Blue at wavelengths $570 \mathrm{~nm}$ and $600 \mathrm{~nm}$ respectively. $\mathrm{R} 1$ and $\mathrm{R} 2=$ molar extinction coefficients of the reduced Alamar Blue at wavelengths $570 \mathrm{~nm}$ and $600 \mathrm{~nm}$ respectively. A1 and A2 = observed absorbance readings for test wells at wavelengths $570 \mathrm{~nm}$ and $600 \mathrm{~nm}$ respectively. $\mathrm{N} 1$ and $\mathrm{N} 2=$ observed absorbance readings for the negative control wells at wavelengths $570 \mathrm{~nm}$ and $600 \mathrm{~nm}$ respectively ${ }^{20}$.

RT-qPCR. Following culture for defined time points, RNA in the 2D culture was extracted using the RNeasy Micro Kit (No. 74004, QIAGEN, Manchester, UK) according to the manufacturer's instructions. An alternative technique for RNA extraction was used for the 3D culture where gels were disrupted and TRIzol (No. 15596026, Thermo Fisher Scientific, Loughborough, UK) added for $10 \mathrm{~min}$. Samples were centrifuged and the RNA in solution transferred to a clean eppendorf. $100 \mu \mathrm{l}$ chloroform was added for $3 \mathrm{~min}$. Samples were centrifuged and the upper aqueous phase transferred to a clean tube. $250 \mu \mathrm{l}$ isopropanol was then mixed in to samples. The eppendorfs were incubated at $-20^{\circ} \mathrm{C}$ for $1 \mathrm{~h}$ and centrifuged. The supernatant was discarded without disrupting the pellet. The RNA pellet was washed twice with $70 \%$ ethanol and centrifuged. The ethanol was removed and the tube air-dried. The pellet was re-suspended in $20 \mu \mathrm{l}$ RNase-free water and incubated at $60^{\circ} \mathrm{C}$ for $10 \mathrm{~min}$. This final incubation facilitates dissolution of the RNA pellet into solution, which otherwise would not be accessible. Heating to $60^{\circ} \mathrm{C}$ for $10 \mathrm{~min}$ for this purpose follows the manufacturer's instruction. In both $2 \mathrm{D}$ and 3D cultures, nucleic acid quantification was performed using a Nanodrop 1000 spectrophotometer. Reading distilled water absorbance acted as a blank, and the samples were read at $260 \mathrm{~nm}$ to give quantification and the ratio of 260/280 used for purity estimation. 100-300 ng of RNA was used to generate cDNA using QuantiTect Reverse Transcription Kit (No. 205311, QIAGEN, Manchester, UK). cDNA was further diluted to a concentration of $5 \mathrm{ng} / \mu \mathrm{l}$ and stored at $-20^{\circ} \mathrm{C}$. RT-qPCR (reverse transcription quantitative polymerase chain reaction) was performed using a SYBR green kit (No. 204143, QIAGEN, Manchester, UK). An Abi7500 thermal cycler (Thermo Fisher Scientific, Loughborough, UK) was used. The primer sequences (Table 1) for the genes were validated by dissociation curve/melt curve analysis. The GapDH (glyceraldehyde 3-phosphate dehydrogenase) housekeeping gene primer was used for normalisation. The $\Delta \mathrm{CT}$ of each sample was calculated with normalisation against GapDH. qRTPCR product quantification was performed using the $2^{-\Delta \Delta C t}$ method. 


\begin{tabular}{|l|l|l|}
\hline Target gene & Forward sequence & Reverse sequence \\
\hline ALP & AGAACCCCAAAGGCTTCTTC & CTTGGCTTTTCCTTCATGGT \\
\hline Cathepsin- $\mathrm{K}$ & GCCAGACAACAGATTTCCATC & CAGAGCAAAGCTCACCACAG \\
\hline CSF-1 & TCCCAGTGATAGAGCCCAGT & CAGGGTCCAGTGAGGTGATG \\
\hline IL- 6 & GATGAGTACAAAAGTCCTGATCCA & CTGCAGCCACTGGTTCTGT \\
\hline M-CSF & GAACTGCCAGTGTAGAGGGAAT & GCTGGTCAGACAACATCTGG \\
\hline NFATc1 & CACCAAAGTCCTGGAGATCCCA & TTCTTCCTCCCGATGTCCGTCT \\
\hline OSCAR & CCAGCTCTAGCGGGTATCTG & GACGGAGTGATGTCTGTGTGAC \\
\hline Osterix & GGCAAAGCAGGCACAAAGAAAG & AATGAGTGGGAAAAGGGAGGG \\
\hline OPN & AGCTGGATGACCAGAGTGCT & TGAAATTCATGGCTGTGGAA \\
\hline OPG & GAAGGGCGCTACCTTGAGAT & GCAAACTGTATTTCGCTCTGG \\
\hline Piezo 1 & CCTGGAGAAGACTGACGGCTAC & ATGCTCCTTGGATGGTGAGTCC \\
\hline RANK & GCTGTAACAAATGTGAACCAGGA & GCCTTGCCTGTATCACAAACT \\
\hline RANKL & TGATTCATGTAGGAGAATTAAACAGG & GATGTGCTGTGATCCAACGA \\
\hline TNF- $\alpha$ & CAGCCTCTTCTCCTTCCTGAT & GCCAGAGGGCTGATTAGAGA \\
\hline TRAP & GGACTGAAGGGACTCCTGAAT & GGTCCCTGAGCCTTTATTCC \\
\hline
\end{tabular}

Table 1. Forward and reverse primer sequences for RT-qPCR.

ELISA. The supernatant was removed from culture wells and stored pending analysis at $-80{ }^{\circ} \mathrm{C}$. The media was last changed $72 \mathrm{~h}$ prior to removal for analysis. ELISA plates were prepared according to the manufacturer's instructions (IL-6 No. DY206, TNF- $\alpha$ No. DY201, OPG No. DY805, R\&D Systems, Abingdon, UK). Plates were then washed and blocked with reagent diluent. A further wash was performed before adding samples and standards. The detection antibody was then added before washing. Streptavidin-HRP was added, avoiding direct light exposure. A further wash was performed. Substrate solution was added for $20 \mathrm{~min}$, avoiding direct light exposure. Finally, stop solution was added. The optical density was calculated at $450 \mathrm{~nm}$ using a Multiskan FC Microplate Photometer. A standard curve was created with Prism v6 and the results interpolated from this.

Osteoclast functional assessment. The $\mathrm{CD} 14^{+}$cell culture was seeded at a concentration of $1 \times 10^{6} / \mathrm{ml}$ onto a 24 well Osteo Assay Surface plate (No. 3987, Corning, Flintshire, UK). RANKL was added at a concentration of $25 \mathrm{ng} / \mathrm{ml}$ after approximately $18 \mathrm{~h}$ incubation. After 7 days incubation, the cell layer was removed by adding $10 \%$ chlorine solution for $10 \mathrm{~min}$. The wells were rinsed before leaving to dry. Images were then captured using an $\operatorname{EVOS}^{\circ}$ FL Auto Cell Imaging System and the area of resorption calculated.

Metabolomics. All the media was removed from the wells before washing with chilled $1 \times$ PBS. $500 \mu \mathrm{l} /$ well of chilled extraction solvent (1:3:1 chloroform: methanol: water) was used. Plates were then sealed with parafilm and vigorously agitated on a rotary shaker for $1 \mathrm{~h}$ at $4{ }^{\circ} \mathrm{C}$. The solvent was removed from the wells and placed in an eppendorf before centrifuging at $1300 \mathrm{rpm}$ for $3 \mathrm{~min}$. The supernatant was removed and placed in a new eppendorf. $50 \mu \mathrm{l}$ of each sample was placed in a separate tube to produce a pooled sample of each condition. Liquid chromatography-mass spectrometry (LC-MS) was performed using the UltiMate 3000 RSLC and Orbitrap Q-Exactive (Thermo Fisher Scientific, Loughborough, UK). The data produced by LC-MS was converted to an IDEOM file. This Excel file details the varying metabolites identified and their respective quantities. Furthermore, the metabolites are linked to the KEGG database (Kyoto Encyclopaedia of Genes and Genomes) which facilitates examination of varying chemicals and biological pathways ${ }^{59}$. The files were generated containing the metabolite KEGG IDs and their ratio to the specified control. These files were analysed with IPA (ingenuity pathway analysis); a programme that identifies and predicts alterations in pathways and networks. No KEGG images or pathways were presented in this study, only IPA networks.

Statistical analysis. The appropriate statistical analyses were preformed using GraphPad Prism software (version 6). Normally distributed data were analysed with the t-test. Conversely, if the data were not normally distributed the Mann-Whitney $U$ test was utilised. Statistically significant results were defined as those having a $p$ value of 0.05 or less.

Received: 2 March 2021; Accepted: 2 November 2021

Published online: 23 November 2021

\section{References}

1. Wade, S. W., Strader, C., Fitzpatrick, L. A., Anthony, M. S. \& O’Malley, C. D. Estimating prevalence of osteoporosis: Examples from industrialized countries. Arch. Osteoporos. 9, 182 (2014).

2. Willson, T., Nelson, S. D., Newbold, J., Nelson, R. E. \& LaFleur, J. The clinical epidemiology of male osteoporosis: A review of the recent literature. Clin. Epidemiol. 7, 65-76 (2015). 
3. Hernlund, E. et al. Osteoporosis in the European Union: medical management, epidemiology and economic burden: A report prepared in collaboration with the International Osteoporosis Foundation (IOF) and the European Federation of Pharmaceutical Industry Associations (EFPIA). Arch. Osteoporos. 8, 136 (2013).

4. Svedbom, A. et al. Osteoporosis in the European Union: A compendium of country-specific reports. Arch. Osteoporos. 8, 137 (2013).

5. Parfitt, A. M. Osteonal and hemi-osteonal remodeling: The spatial and temporal framework for signal traffic in adult human bone. J. Cell Biochem. 55, 273-286 (1994).

6. Tu, K. N. et al. Osteoporosis: A review of treatment options. J. Cell Biochem. 43, 92-104 (2018).

7. Rodriguez, J. P., Rios, S., Fernandez, M. \& Santibanez, J. F. Differential activation of ERK1,2 MAP kinase signaling pathway in mesenchymal stem cell from control and osteoporotic postmenopausal women. J. Cell. Biochem. 92, 745-754 (2004).

8. Mizuno, A. et al. Severe osteoporosis in mice lacking osteoclastogenesis inhibitory factor/osteoprotegerin. Biochem. Biophys. Res. Commun. 247, 610-615 (1998).

9. Owen, R. \& Reilly, G. C. In vitro models of bone remodelling and associated disorders. Front. Bioeng. Biotechnol. 6, 134 (2018).

10. Komori, T. Animal models for osteoporosis. Eur. J. Pharmacol. 759, 287-294 (2015).

11. Turner, R. T. et al. Animal models for osteoporosis. Rev. Endocr. Metab. Disord. 2, 117-127 (2001).

12. Zhao, S., Zhang, Y. K., Harris, S., Ahuja, S. S. \& Bonewald, L. F. MLO-Y4 osteocyte-like cells support osteoclast formation and activation. J. Bone Miner. Res. 17, 2068-2079 (2002).

13. Chen, S. T. et al. (-)-Epigallocatechin-3-gallate decreases osteoclastogenesis via modulation of RANKL and osteoprotegrin. Molecules 24, 1-156 (2019).

14. Hayden, R. S., Quinn, K. P., Alonzo, C. A., Georgakoudi, I. \& Kaplan, D. L. Quantitative characterization of mineralized silk film remodeling during long-term osteoblast-osteoclast co-culture. Biomaterials 35, 3794-3802 (2014).

15. Schulze, S., Wehrum, D., Dieter, P. \& Hempel, U. A supplement-free osteoclast-osteoblast co-culture for pre-clinical application. J. Cell Physiol. 233, 4391-4400 (2018).

16. Kim, C. H., You, L., Yellowley, C. E. \& Jacobs, C. R. Oscillatory fluid flow-induced shear stress decreases osteoclastogenesis through RANKL and OPG signaling. Bone 39, 1043-1047 (2006).

17. Young, P. S., Tsimbouri, P. M., Gadegaard, N., Meek, R. M. \& Dalby, M. J. Osteoclastogenesis/osteoblastogenesis using human bone marrow-derived cocultures on nanotopographical polymer surfaces. Nanomedicine 10, 949-957 (2015).

18. Silverwood, R. K. et al. Analysis of osteoclastogenesis/osteoblastogenesis on nanotopographical titania surfaces. Adv. Healthc. Mater. 5, 947-955 (2016).

19. Nikukar, H. et al. Osteogenesis of mesenchymal stem cells by nanoscale mechanotransduction. ACS Nano 7, 2758-2767 (2013).

20. Tsimbouri, P. M. et al. Stimulation of 3D osteogenesis by mesenchymal stem cells using a nanovibrational bioreactor. Nat. Biomed. Eng. 1, 758-770 (2017).

21. Robertson, S. N. et al. Control of cell behaviour through nanovibrational stimulation: Nanokicking. Philos. Trans. A Math. Phys. Eng. Sci. 28, 2120 (2018).

22. Orapiriyakul, W. et al. Nanovibrational stimulation of mesenchymal stem cells induces therapeutic reactive oxygen species and inflammation for three-dimensional bone tissue engineering. ACS Nano 14, 10027-10044 (2020).

23. Campsie, P. et al. Design, construction and characterisation of a novel nanovibrational bioreactor and cultureware for osteogenesis. Sci. Rep. 9, 12944 (2019).

24. Engler, A. J., Sen, S., Sweeney, H. L. \& Discher, D. E. Matrix elasticity directs stem cell lineage specification. Cell 126, 677-689 (2006).

25. Kell, G. S. \& Whalley, E. The PVT properties of water. Philos. Trans. A. Math. Phys. Eng. Sci. 258, 565-617 (1965).

26. Kim, J. H. et al. Role of CrkII signaling in RANKL-induced osteoclast differentiation and function. J. Immunol. 196, 1123-1131 (2016).

27. Choy, E. H. et al. Translating IL-6 biology into effective treatments. Nat. Rev. Rheumatol. 16, 335-345 (2020).

28. Kang, J. H. et al. Osteoprotegerin expressed by osteoclasts: an autoregulator of osteoclastogenesis. J. Dent. Res. 93, 1116-1123 (2014).

29. Abu-Amer, Y. NF-kappaB signaling and bone resorption. Osteoporos. Int. 24, 2377-2386 (2013).

30. Boyce, B. F., Xiu, Y., Li, J., Xing, L. \& Yao, Z. NF-kappaB-mediated regulation of osteoclastogenesis. Endocrinol. Metab. 30, 35-44 (2015).

31. Xie, L. et al. Low-level mechanical vibrations can influence bone resorption and bone formation in the growing skeleton. Bone 39, 1059-1066 (2006).

32. Meng, J. et al. Low-intensity pulsed ultrasound inhibits RANKL-induced osteoclast formation via modulating ERK-c-Fos-NFATc1 signaling cascades. Am. J. Transl. Res. 10, 2901-2910 (2018).

33. Wang, L. et al. Mechanical sensing protein PIEZO1 regulates bone homeostasis via osteoblast-osteoclast crosstalk. Nat. Commun. 11, 282 (2020).

34. McNamara, L. E. et al. Skeletal stem cell physiology on functionally distinct titania nanotopographies. Biomaterials 32, 7403-7410 (2011).

35. Tsimbouri, P. M. et al. Using nanotopography and metabolomics to identify biochemical effectors of multipotency. ACS Nano 6, 10239-10249 (2012).

36. Roberts, J. N. et al. Dynamic surfaces for the study of mesenchymal stem cell growth through adhesion regulation. ACS Nano 10, 6667-6679 (2016).

37. Alakpa, E. V. et al. Tunable supramolecular hydrogels for selection of lineage-guiding metabolites in stem cell cultures. Chem $\mathbf{1}$, 298-319 (2016).

38. Hodgkinson, T. et al. The use of nanovibration to discover specific and potent bioactive metabolites that stimulate osteogenic differentiation in mesenchymal stem cells. Sci. Adv. 7, 9 (2021).

39. Brey, D. M. et al. High-throughput screening of a small molecule library for promoters and inhibitors of mesenchymal stem cell osteogenic differentiation. Biotechnol. Bioeng. 108, 163-174 (2011).

40. Kawamura, N. et al. Akt1 in osteoblasts and osteoclasts controls bone remodeling. PLoS ONE 2, e1058 (2007).

41. Amini, A. R., Laurencin, C. T. \& Nukavarapu, S. P. Bone tissue engineering: Recent advances and challenges. Crit. Rev. Biomed. Eng. 40, 363-408 (2013).

42. Ahlmann, E., Patzakis, M., Roidis, N., Shepherd, L. \& Holtom, P. Comparison of anterior and posterior iliac crest bone graft in terms of harvest-site morbidity and functional outcomes. J. Bone Joint Surg. Am. 84, 716-720 (2002).

43. Dimitriou, R., Mataliotakis, G. I., Angoules, A. G., Kanakaris, N. K. \& Giannoudis, P. V. Complications following autologous bone gra harvesting from the iliac crest and using the RIA: A systematic review. Injury 42, S3-S15 (2011).

44. Carragee, E. J. et al. Cancer risk after use of recombinant bone morphogenetic protein-2 for spinal arthrodesis. J. Bone Joint Surg. Am. 95, 1537-1545 (2013).

45. Raida, M. et al. Bone morphogenetic protein 2 (BMP-2) and induction of tumor angiogenesis. J. Cancer Res. Clin. Oncol. 131, $741-750$ (2005).

46. Olson, J. L., Atala, A. \& Yoo, J. J. Tissue engineering: current strategies and future directions. Chonnam Med. J. 47, 1-13 (2011).

47. Tang, P., Xiong, Q., Ge, W. \& Zhang, L. The role of microRNAs in osteoclasts and osteoporosis. RNA Biol. 11, 1355-1363 (2014). 
48. Saftig, P. et al. Impaired osteoclastic bone resorption leads to osteopetrosis in cathepsin-K-deficient mice. Proc. Natl. Acad. Sci. USA 95, 13453-13458 (1998).

49. Naito, A. et al. Severe osteopetrosis, defective interleukin-1 signalling and lymph node organogenesis in TRAF6-deficient mice. Genes Cells 4, 353-362 (1999).

50. Wang, W. \& Yeung, K. W. K. Bone grafts and biomaterials substitutes for bone defect repair: A review. Bioact. Mater. 2, 224-247 (2017).

51. White, S. L. et al. The global diffusion of organ transplantation: trends, drivers and policy implications. Bull. World Health. Organ. 92, 826-835 (2014).

52. Vanleene, M. \& Shefelbine, S. J. Therapeutic impact of low amplitude high frequency whole body vibrations on the osteogenesis imperfecta mouse bone. Bone 53, 507-514 (2013).

53. Fischer, M. et al. Long-term effects of whole-body vibration on human gait: A systematic review and meta-analysis. Front. Neurol. 10, 627 (2019).

54. Nagasaki, R. et al. A combination of low-intensity pulsed ultrasound and nanohydroxyapatite concordantly enhances osteogenesis of adipose-derived stem cells from buccal fat pad. Cell Med. 7, 123-131 (2015).

55. Feng, L. et al. A comparison of 1- and 3.2-MHz low-intensity pulsed ultrasound on osteogenesis on porous titanium alloy scaffolds: An in vitro and in vivo study. J. Ultrasound Med. 38, 191-202 (2019).

56. Dionello, C. F. et al. Effects of whole body vibration exercises on bone mineral density of women with postmenopausal osteoporosis without medications: Novel findings and literature review. J. Musculoskelet. Neuronal Interact. 16, 193-203 (2016).

57. Leighton, R. et al. Healing of fracture nonunions treated with low-intensity pulsed ultrasound (LIPUS): A systematic review and meta-analysis. Injury 48, 1339-1347 (2017).

58. May, K. R. \& Walker, B. N. The effects of distractor sounds presented through bone conduction headphones on the localization of critical environmental sounds. Appl. Ergon. 61, 144-158 (2017).

59. Fiehn, O. Metabolomics: The link between genotypes and phenotypes. Plant Mol. Biol. 48, 155-171 (2002).

\section{Acknowledgements}

The authors would like to thank C.A. Smith for contributing to this study. The authors also acknowledge the support of NHS research Scotland (NRS) Greater Glasgow and Clyde Bio-repository.

\section{Author contributions}

J.K., M.T., P.C. and S.S. contributed to study design and experimental data collection. P.G.C., S.R. and M.D. contributed to design of nanovibrational bioreactor. M.D. also contributed to study design and production of figures. P.Y. and C.G. contributed to study design and methodology. D.M. contributed to cell harvesting. The manuscript was written through contributions of all authors. All authors have given approval to the final version of the manuscript.

\section{Funding}

This work was funded by The Engineering and Physical Sciences Research Council (grant number EP/ N013905/1) \& The Royal College of Surgeons Edinburgh (grant number SPPG/17/113).

\section{Competing interests}

The authors declare no competing interests.

\section{Additional information}

Supplementary Information The online version contains supplementary material available at https://doi.org/ 10.1038/s41598-021-02139-9.

Correspondence and requests for materials should be addressed to J.W.K.

Reprints and permissions information is available at www.nature.com/reprints.

Publisher's note Springer Nature remains neutral with regard to jurisdictional claims in published maps and institutional affiliations.

(c) (i) Open Access This article is licensed under a Creative Commons Attribution 4.0 International (c) License, which permits use, sharing, adaptation, distribution and reproduction in any medium or format, as long as you give appropriate credit to the original author(s) and the source, provide a link to the Creative Commons licence, and indicate if changes were made. The images or other third party material in this article are included in the article's Creative Commons licence, unless indicated otherwise in a credit line to the material. If material is not included in the article's Creative Commons licence and your intended use is not permitted by statutory regulation or exceeds the permitted use, you will need to obtain permission directly from the copyright holder. To view a copy of this licence, visit http://creativecommons.org/licenses/by/4.0/.

(C) The Author(s) 2021 